\title{
Peak amplitude of target current determines deposition rate loss during high power pulsed magnetron sputtering
}

\author{
Grzegorz Greczynski and Lars Hultman
}

\section{Linköping University Post Print}

\section{Tweet}

N.B.: When citing this work, cite the original article.

Original Publication:

Grzegorz Greczynski and Lars Hultman, Peak amplitude of target current determines deposition rate loss during high power pulsed magnetron sputtering, 2016, Vacuum, (124).

http://dx.doi.org/10.1016/j.vacuum.2015.11.004

Copyright: Elsevier

$$
\text { http://www.elsevier.com/ }
$$

Postprint available at: Linköping University Electronic Press

http://urn.kb.se/resolve?urn=urn:nbn:se:liu:diva-125682

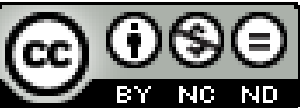




\title{
Peak amplitude of target current determines deposition rate loss during high power pulsed magnetron sputtering
}

\author{
G. Greczynski and L. Hultman \\ Thin Film Physics Division, Department of Physics (IFM), Linköping University, \\ SE-581 83 Linköping, Sweden
}

\begin{abstract}
Film growth rates during DCMS and HIPIMS sputtering in Ar are measured for ten technologically-relevant elemental target materials: Al, Si, Ti, Cr, Y, Zr, Nb, Hf, Ta, and W, spanning wide range of masses, ionization energies, and sputter yields. Surprisingly, the ratio of power-normalized HIPIMS and DCMS rates $\alpha$ decays exponentially with increasing peak target current density $J_{T}^{\max }$ for all metals. The effect of $J_{T}^{\max }$ on $\alpha$ is dramatic: $\alpha \approx 1$ in the limit of lowest $J_{T}^{\max }$ values tested $\left(0.04 \mathrm{~A} / \mathrm{cm}^{2}\right)$ and decreases to only 0.12 with $J_{T}^{\max } \sim 3 \mathrm{~A} / \mathrm{cm}^{2}$. With the exception of $\mathrm{Al}$ and $\mathrm{Si}, \alpha\left(J_{T}^{\max }\right)$ curves overlap indicating that the debated rate loss in HIPIMS is to large extent determined by the peak amplitude of the HIPIMS target current for all tested metals. Back attraction of ionized target species is responsible for such large variation in $\alpha$.
\end{abstract}

Keywords: HIPIMS; growth rate; deposition rate; magnetron sputtering, ionized PVD 
High power pulsed magnetron sputtering (HIPIMS or HPPMS) ${ }^{1}$ is a valuable addition to magnetron-sputtering-based PVD techniques. ${ }^{2,3,4,5}$ HIPIMS is particularly attractive for the growth of transition metal (TM) nitride layers for two main reasons: (i) the ability to ionize up to $90 \%$ of the sputtered metal flux with an option to control the ionization degree by varying the peak target current density, ${ }^{6,7}$ and (ii) the time separation of metal- and gas-ion fluxes from the target. ${ }^{8}$ The former effect is ascribed to the effective electron-impact ionization in the highdensity plasma region ${ }^{9}$ formed, for a short period of time, 20-100 $\mu$ s, in front of the sputtering target operated in HIPIMS mode. The latter effect, caused by a strong gas rarefaction taking place during the high-power pulse $\mathrm{e}^{10,11,12}$ allows the control of metal-ion momentum and energy arriving onto a growing film, through the synchronization of the substrate bias pulse to the metal-ion rich part of the sputter-deposited flux, ${ }^{13,14}$ with decisive influence on film nanostructure, phase content, and stress state. ${ }^{15,16}$

It was postulated early that the benefits of HIPIMS in terms of high ionization of the target material come at the expense of a lowered deposition rate, ${ }^{17,18,19,20}$ implying worse process economics which is currently a perceived obstacle for industrial implementation. Numerous phenomena have been proposed to account for this behavior including (i) the socalled "return effect" - an effective capturing of the ionized portion of the sputter-ejected flux by the cathode field, ${ }^{21,22}$ (ii) an enhanced azimuthal ion transport (across the magnetic field lines), ${ }^{23}$ (iii) a “yield effect” since usually HIPIMS operates at higher target voltage $V_{T}$ than in conventional DC magnetron sputtering (DCMS) one may expect lower power-normalized rate due to the fact that sputter yields are not directly proportional to $V_{T},{ }^{24}$ and (iv) "species effect" related to the change of effective sputter yield once gas ions are replaced with the back-attracted target ions in the later phase of the pulse. ${ }^{25}$

The reported values for the loss of power-normalized HIPIMS deposition rate $\bar{R}_{\text {HIPIMS }}$ show large spread even for the same material system. For example, if referred to the power- 
normalized DCMS rate $\bar{R}_{D C M S}$, the ratio $\bar{R}_{\text {HIPIMS }} / \bar{R}_{D C M S}$ varies from $15 \%$ to $75 \%$ for Ti, and from $37 \%$ to $80 \%$ in the case of $\mathrm{Cu}^{2,26}$ This may be due to different HIPIMS settings and prevents systematic comparisons between material systems, as well as, identification of key parameters for the here contested effect of HIPIMS rate loss.

We performed film growth rate measurements under identical conditions for ten elemental targets representing widely different masses, melting temperatures, sputtering yields, ionization potentials, and secondary electron emission yields during HIPIMS and DCMS in Ar atmosphere. The HIPIMS peak target current density $J_{T}^{\max }$ is varied by two orders of magnitude, from 0.04 to $3 \mathrm{~A} / \mathrm{cm}^{2}$, by means of changing the average power settings at constant pulsing frequency. We find that materials that are prone to high target current densities during HIPIMS discharge have second ionization potential $I P_{T}^{2}$ lower than the first ionization potential of $\mathrm{Ar}$ $I P_{A r}^{1}$. This results in higher concentrations of doubly-ionized sputter-ejected species that, in turn, enable effective potential emission of secondary electrons during the metal (selfsputtering) phase and, hence, enhanced ionization of sputtering gas. For all materials, the ratio of power normalized HIPIMS and DCMS rates $\alpha$ decays exponentially with increasing peak target current density $J_{T}^{\max }$. Surprisingly, with the exception of $\mathrm{Al}$ and $\mathrm{Si}, \alpha\left(J_{T}^{\max }\right)$ curves overlap indicating that the rate loss in HIPIMS is to large extent determined by the peak amplitudes of the HIPIMS target currents.

Aluminum, Si, Ti, Cr, Y, Zr, Nb, Hf, Ta, and W films are grown in a CC800/9 CemeCon AG magnetron sputtering system using rectangular $8.8 \times 50 \mathrm{~cm}^{2}$ targets that are virgin to exclude possible influence of the target history. The same magnetron is used in the experiments to ensure the same magnetic field strength and distribution. Prior to film growth each target undergoes a sputter-in sequence consisting of (1) DCMS operation with the average power $\langle P\rangle_{T}$ increasing from 1 to $8 \mathrm{~kW}$ in steps of $1 \mathrm{~kW}$ and 5 min per step, followed by (2) $1 \mathrm{~h}$ DCMS with $\langle P\rangle_{T}=4 \mathrm{~kW}$, and (3) HIPIMS with average power set to $2 \mathrm{~kW}$ and a frequency $f$ of $1000 \mathrm{~Hz}$ 
resulting in rather low target current density values which increase as $f$ is gradually decreased to 500, 300, 200, and $100 \mathrm{~Hz}$. Finally, frequency is set to $200 \mathrm{~Hz}$ (as for the film growth) and $\langle P\rangle_{T}$ is scanned from 0.4 to $4 \mathrm{~kW}$ during less than $15 \mathrm{~min}$ in order to assure stable operation in a wide current density range.

Si(001) substrates, $2 \times 1 \mathrm{~cm}^{2}$, are cleaned sequentially in acetone and isopropyl alcohol, and mounted at a distance of only $6 \mathrm{~cm}$ from the target to minimize the influence of gas scattering. ${ }^{27}$ The growth chamber is degassed prior to deposition during a 2-h-long heating cycle so that the system base pressure is $2.3 \times 10^{-6}$ Torr $(0.3 \mathrm{mPa})$. Targets are operated in pure Ar with the gas flow $f_{A r}$ set at $420 \mathrm{~cm}^{3} / \mathrm{min}$, resulting in the total pressure of $3 \mathrm{mTorr}(0.4 \mathrm{~Pa})$. The substrate temperature is $400{ }^{\circ} \mathrm{C}$.

Two multilayer films are grown for each material system, one in HIPIMS and one in DCMS mode, with the average target power $\langle P\rangle_{T}$ varied from 0.4 to $4 \mathrm{~kW}$ in steps of $0.4 \mathrm{~kW}$. During HIPIMS experiments the pulsing frequency $f$ is fixed at $200 \mathrm{~Hz}$, thus variation in $\langle P\rangle_{T}$ corresponds to changing the pulse energy $E_{p}$ from 2 to $20 \mathrm{~J}$ in steps of $2 \mathrm{~J}\left(\langle P\rangle_{T}\right.$ and $E_{p}$ are related by $\langle P\rangle_{T}=f \times E_{p}$ ). While the preset pulse length $T_{O N}$ is $200 \mu \mathrm{s}$, the effective pulse length $\tau$, defined here as the time period during which $J_{T}(t)>0.1 \times J_{T}^{\max }$, may vary depending on $\langle P\rangle_{T}$ and target material. Resulting film thickness of individual layers is in the range from 350 to $650 \mathrm{~nm}$. A 50-nm-thick TiN marker layers are deposited after each step in order to facilitate the layer thickness determination by cross-sectional scanning electron microscopy (XSEM) analyses. For the TiN growth in mixed $\mathrm{Ar} / \mathrm{N}_{2}$ atmosphere, the target is protected by cathode shutters to avoid cross-contamination from the neighboring cathode equipped with Ti target. All tested targets are pre-sputtered behind close shutters to remove possibly formed surface nitride layer following each TiN deposition step. A negative dc substrate bias, $V_{s}=-60$ $\mathrm{V}$ is used during DCMS film growth, while pulsed bias voltage with the same amplitude and 4 \% duty cycle, synchronized with the HIPIMS pulse, is used for HIPMS experiments. ${ }^{13,14}$ 
Target current $I_{T}(t)$ and target voltage $V_{T}(t)$ waveforms (in which $T$ stands for the target material) during film growth are recorded with a Tektronix $500 \mathrm{MHz}$ bandwidth digital oscilloscope. The target current density $J_{T}(t)$, a more generic quantity, is obtained by dividing $I_{T}(t)$ with the entire target area $A=440 \mathrm{~cm}^{2}$.

Figures $1(\mathrm{a}-\mathrm{d})$ show $J_{T}(t)$ and $V_{T}(t)$ waveforms recorded with the pulse energy $E_{p}=18$ J during the HIPIMS operation of different metal targets in Ar. Data presented reveal large differences with respect to current-voltage performance between the materials. Based on the appearance of $J_{T}(t)$ and $V_{T}(t)$ curves as well as their evolution with $E_{p}$ (not shown) the target materials can be divided into two categories. The first category, including Ti, Zr, Nb, and Y (Figs. 1(a) and 1(c)), is characterized by narrow, high-amplitude current pulses, and relatively short triangular voltage waveforms that posses low amplitudes towards the end of the HIPIMS pulse. ${ }^{28}$ For materials in the second category ( $\mathrm{Si}, \mathrm{Cr}, \mathrm{Hf}$, Ta, and $\mathrm{W}$ ), current pulses are relatively broad with low-amplitudes, while $V_{T}(t)$ traces are characterized by higher voltage values towards the end of the pulse. Al target constitutes an intermediate case between category I and II metals. Trends outlined here are independent of the average power setting.

In the case of category I metals the peak target current density $J_{T}^{\max }$ reaches $2.4 \mathrm{~A} / \mathrm{cm}^{2}$ for $\mathrm{Zr}$ and $\mathrm{Nb}, 3 \mathrm{~A} / \mathrm{cm}^{2}$ for $\mathrm{Ti}$, and $3.4 \mathrm{~A} / \mathrm{cm}^{2}$ for $\mathrm{Y}$. The effective pulse lengths $\tau$ is in the range from 51 to $59 \mu \mathrm{s}$. As a consequence of these extremely high currents PS capacitors get almost completely emptied resulting in low $V_{T}$ values towards the end of the pulse (cf. Fig. 1(c)). Materials in this category are characterized by rather low second ionization potential $I P_{T}^{2}$ values (cf. Tab.1), all satisfying the criterion $I P_{T}^{2}<I P_{A r}^{1}\left(I P_{A r}^{1}=15.76 \mathrm{eV}\right)^{29}$ meaning that there is a significant electron population in the discharge with energies in the range $I P_{T}^{2}<E_{e}<I P_{A r}^{1}$, i.e., too low to ionize Ar, yet high enough to produce doubly-ionized target ions. ${ }^{30}$ This is of key importance in HIPIMS plasmas, since the primary ionization mechanism is by electron impact. As pointed out by Anders et al. ${ }^{31}$ the presence of higher charge state species is essential 
for the secondary electron emission during the metal-dominated (self-sputtering) phase of the HIPIMS pulse, when concentrations of inert gas ions is lowered due to rarefaction effects. ${ }^{10,11,12}$ For relatively low ion energy used in sputtering, secondary electrons are predominantly ejected by potential emission, which requires that the condition: ${ }^{32}$

$$
0.39 I P>\phi_{T}
$$

in which $\phi_{T}$ is the target work function, ${ }^{33}$ is satisfied. It follows from data presented in Table 1 that all $I P_{T}^{1}$ values are too low to fulfill Eqn.(1) and doubly-charged species are necessary for the potential emission to set in, in the absence of gas ions. Eqn.(1) is readily satisfied in the case of category I metals, for which higher concentrations of doubly-ionized species are indeed expected, leading to effective secondary electron emission during the metal (self-sputtering) phase, resulting in thorough ionization of the working gas (diluted due to rarefaction) and high target current densities.

In distinct contrast, for category II metals operation with $E_{p}=18 \mathrm{~J}$ results in relatively low $J_{T}^{\max }$ values, from $0.9 \mathrm{~A} / \mathrm{cm}^{2}$ for $\mathrm{W}$ to $1.3 \mathrm{~A} / \mathrm{cm}^{2}$ for Ta target. Pulses become broader with $\tau$ values 78-79 $\mu$ s for $\mathrm{Cr}$ and $\mathrm{Ta}$, to 84,105 , and $106 \mu$ s with $\mathrm{Si}, \mathrm{Hf}$, and $\mathrm{W}$, respectively. In addition, $J_{T}(t)$ waveforms exhibit a more complex form (in opposite to category I metals) characterized by a fast rise of the current density during the first $10 \mu$ s followed by a short, $~ 5$ $\mu$ s, plateau-like period (best visible for $\mathrm{W}, \mathrm{Hf}$, and $\mathrm{Cr}$ ) after which $J_{T}(t)$ continues to rise until the $J_{T}^{\max }$ is reached. Materials $\mathrm{Cr}, \mathrm{W}$ or $\mathrm{Hf} J_{T}(t)$ also exhibit a pronounced shoulder at $t \sim 60$ $\mu$ s. Capacitors do not get fully discharged due to lower target current, hence $V_{T}$ stays relatively high towards the end of the HIPIMS pulse (cf. Fig. 1(d)). Contrary to category I metals, the majority of category II metals has $I P_{T}^{2}$ either greater or similar to $I P_{A r}^{1}$, hence lower concentrations of doubly-ionized species are expected. The secondary electron emission is thus predominantly due to noble gas ions. Once the $\mathrm{Ar}^{+}$concentration decreases due to rarefaction 
so does the electron emission, hence the resulting target current density gets seriously limited during the self-sputtering phase.

Target current density is surprisingly high for the $\mathrm{Al}$ target that possesses highest $I P_{T}^{2}$ in the whole set (see Tab. 1) resulting in negligible $\mathrm{Al}^{2+}$ fluxes. ${ }^{34}$ One reason may be relatively low melting point $\left(660^{\circ} \mathrm{C}\right)$ of $\mathrm{Al}$, such that the target surface is at the onset of evaporation during most energetic phase of HIPIMS discharge. ${ }^{35}$ The film growth rate measurements presented below are in favor of this interpretation.

HIPIMS film growth rates are commonly compared to corresponding DCMS rates obtained at the same average target power. ${ }^{25}$ Therefore, in Figure 2 the ratio $\alpha=$ $\bar{R}_{\text {HIPIMS }} / \bar{R}_{D C M S}$ of power-normalized rates is plotted as a function of peak target current density for all target materials tested. Clearly, the loss of deposition rate with increasing $J_{T}^{\max }$ takes place for each metal, irrespective of target ability to produce high current densities (category I vs. category II metals). In the limit of lowest $J_{T}^{\max }$ values, $\alpha\left(J_{T}^{\max }\right)$ curves approach unity indicating nearly-DCMS sputter efficiency, which means that pulsing itself has no effect on the rate drop. With increasing peak target current density $\alpha$ exhibits nearly exponential decay for all materials. Interestingly, with the exception for $\mathrm{Al}$ and $\mathrm{Si}$, all $\alpha\left(J_{T}^{\max }\right)$ curves nearly overlap such that for any $J_{T}^{\max }$ value the spread in $\alpha$ is only $+/-5 \%$, and even less than that in the high current limit, $J_{T}^{\max }>1.5 \mathrm{~A} / \mathrm{cm}^{2}$ (category I). This shows that the rate loss in HIPIMS is to a large extent determined by the peak amplitude of the HIPIMS target current in a wide $J_{T}^{\max }$ range spanning nearly two orders of magnitude, from 0.04 to $3 \mathrm{~A} / \mathrm{cm}^{2}$. There is no apparent difference between category I and category II metals in this respect.

In order to evaluate the potential contribution of the "yield effect" ${ }^{24}$ to the observed loss of deposition rate we calculated the ratio of time-averaged power-normalized deposition rates $\overline{\langle R\rangle}_{\text {HIPIMS }} / \overline{\langle R\rangle}_{D C M S}$ accordingly to ${ }^{36}$ 


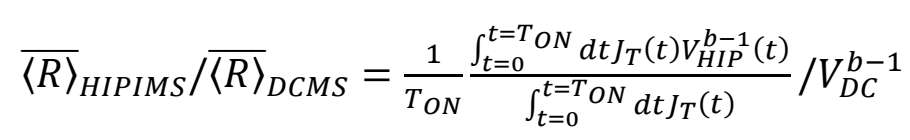

in which $b$ is a constant for the target material obtained by fitting sputter yield $\gamma_{A r \rightarrow M}$ values calculated for a range of incident ion energies $E_{i}$ using TRIM (Transport of Ions in Matter) software package, ${ }^{37,38}$ with an allometric function of the form $a E_{i}^{b}$. Eqn.(2) was evaluated for each power setting and target material using the recorded current density and voltage functions and the results compared with the experimental observations in Fig.2. We find that $\overline{\langle R\rangle}_{\text {HIPIMS }} / \overline{\langle R\rangle}_{D C M S}$ ranges from 0.75 for $\mathrm{Cr}$ to 0.97 for $\mathrm{Nb}$ and shows only a weak dependence on $J_{T}^{\max }$. Thus, the "yield effect" does not account for the observed exponential decay in $\alpha$, which decreases to values as low as 0.1 in the limit of highest $J_{T}^{\max }$.

Potential influence of the "return effect", as well as, the "species effect" on $\alpha$ is treated explicitly by the target materials pathway model ${ }^{21}$. Within this approach

$$
\alpha=\frac{1-\beta+\beta(1-\sigma) \eta_{T}}{1+\beta \sigma\left(\gamma_{A r \rightarrow M}-\gamma_{M \rightarrow M}\right)}
$$

in which $\beta$ is the ionized fraction of the sputtered flux, $\sigma$ is the fraction of the ionized metal species that gets captured by the magnetron and directed to the target for sputtering, $\eta_{T}$ is the fraction of sputtered species that is available for deposition (assuming that the transport from the target to the substrate is the same as during DCMS), finally $\gamma_{A r \rightarrow M}$ and $\gamma_{M \rightarrow M}$ are sputtering and self-sputtering yields. While it is unknown how $\sigma$ and $\eta_{T}$ vary with increasing $J_{T}^{\max }$, it is commonly accepted that the ionization of the sputtered flux (hence $\beta$ ) increases with increasing peak target current density due to higher probability for electron-impact ionization. ${ }^{9}$ Thus, the straightforward interpretation of results shown in Fig. 2 is that, $\alpha$ decreases with increasing $J_{T}^{\max }$ for all metals due to increasing $\beta$. In the limit of the lowest $J_{T}^{\max }, \beta \rightarrow 0$ (no or little ionization) and $a \rightarrow 1\left(\bar{R}_{\text {HIPIMS }} \sim \bar{R}_{D C M S}\right)$, as indeed observed. For the highest $J_{T}^{\max }$ values, $\beta \rightarrow 1$ and Eqn.(3) becomes 


$$
\alpha_{\beta \rightarrow 1}=\frac{(1-\sigma) \eta_{T}}{1+\sigma\left(\gamma_{A r \rightarrow M}-\gamma_{M \rightarrow M}\right)}
$$

In the case of $\sigma \approx 1$ ( $100 \%$ capturing efficiency) $\alpha_{\beta \rightarrow 1} \approx 0$ (HIPIMS rate approaches zero), which clearly does not take place in our experiments, where $0.1<\alpha_{\beta \rightarrow 1}<0.15$ (see Fig. 2).

The main explanations for significantly higher $\alpha$ values observed for Al and Si targets are (1) the short plasma transit time of the relatively low-mass sputter-ejected $\mathrm{Al}$ and Si atoms that decreases the probability for electron-impact ionization events resulting in lower ionization (lower $\beta$ in Eqn.(3) implies higher $\alpha$ ), and (2) Al and Si have $\gamma_{A r \rightarrow M}<\gamma_{M \rightarrow M}$ (see Tab.(1)), by which the sputtering rate should increase as the discharge transforms into the metallic phase (assuming constant $\beta$ and $\sigma$ ) with a positive effect on $\alpha$.

In conclusion, $\mathrm{Ti}, \mathrm{Y}, \mathrm{Zr}$, and $\mathrm{Nb}$ yield very high peak target current densities during HIPIMS discharge, not achievable with other targets. This is because their second ionization potentials $I P_{T}^{2}$ are significantly lower than the first ionization potential of Ar $I P_{A r}^{1}$ leading to higher concentrations of doubly-ionized sputter-ejected species in the plasma that cause effective secondary electron emission during the metal (self-sputtering) phase and enhanced ionization of sputtering gas diluted due to rarefaction. For all metals included in this study, the ratio of power normalized HIPIMS and DCMS rates $\alpha$ decays exponentially with increasing peak target current density from $\alpha=1$ in the limit of lowest $J_{T}^{\max }$ tested to $\alpha \sim 0.1$ with $J_{T}^{\max }$ $\sim 3 \mathrm{~A} / \mathrm{cm}^{2}$. Surprisingly, with the exception for $\mathrm{Al}$ and $\mathrm{Si}$, all $\alpha\left(J_{T}^{\max }\right)$ curves overlap indicating that the rate loss in HIPIMS is to large extent determined by the peak amplitude of the HIPIMS target current. The "return effect", i.e., back attraction of ionized target species, is the only mechanism that can account for such large variation in $\alpha$. Hence, the choice of HIPIMS working condition for a particular application, should be based on an optimization between required ionization degree and loss of deposition rate by taking advantage of a wide process parameter space. 
We acknowledge support from the VINN Excellence Center Functional Nanoscale Materials (FunMat) Grant 2005-02666, the Swedish Government Strategic Research Area in Materials Science on Functional Materials at Linköping University (SFO-Mat-LiU 2009-00971), and the Knut and Alice Wallenberg Foundation Scholar Grant 2011.0143. 


\section{Figure and Table Captions}

Fig. 1. (a-b) Target current density $J_{T}(t)$, and (c-d) target voltage $V_{T}(t)$ waveforms recorded during HIPIMS process with pulse energy of $18 \mathrm{~J}$ for $\mathrm{Al}, \mathrm{Si}, \mathrm{Ti}, \mathrm{Cr}, \mathrm{Y}, \mathrm{Zr}, \mathrm{Nb}, \mathrm{Hf}, \mathrm{Ta}$, and $\mathrm{W}$ targets.

Fig. 2. The ratio of HIPIMS and DCMS power-normalized film growth rates $\alpha=$ $\bar{R}_{\text {HIPIMS }} / \bar{R}_{D C M S}$ as a function of peak target current density $J_{T}^{\max }$ for $\mathrm{Al}, \mathrm{Si}, \mathrm{Ti}, \mathrm{Cr}, \mathrm{Y}$, Zr, Nb, Hf, Ta, and W targets operated in HIPIMS mode. Category I and category II metals are indicated in the legend.

Tab. 1 Target material parameters relevant for HIPIMS and DCMS sputtering. Atomic mass $m$, bulk density $\rho$, homologous temperature $T_{s} / T_{m}$, first and second ionization potential $I P_{T}^{1}$ and $I P_{T}^{2},{ }^{29}$ as well as work function $\phi_{T} \cdot{ }^{33}$ Sputter yield for $1 \mathrm{keV}$ Ar ions $\gamma_{A r \rightarrow M}$ as well as self-sputtering yield for $1 \mathrm{keV}$ ions $\gamma_{M \rightarrow M}$ are calculated using TRIM software. $^{38}$ 


\section{REFERENCES}

${ }^{1}$ V. Kouznetsov, K. Macak, J. M. Schneider, U. Helmersson and I. Petrov, Surf. Coat. Technol. 122 (1999) 290

${ }^{2}$ K. Sarakinos, J. Alami, S. Konstantinidis, Surf. Coat. Technol. 204 (2010) 1661-1684

${ }^{3}$ A. Anders, Surf. Coat. Technol. 257 (2014) 308

${ }^{4}$ A. Anders, Surf. Coat. Technol. 204 (2010) 2864

${ }^{5}$ A. Anders, Surf. Coat. Technol. 205 (2011) S1-S9

${ }^{6}$ J. Böhlmark, J. Alami, C. Christou, A.P. Ehiasarian, U. Helmersson, J. Vac. Sci. Technol. A 23 (2005) 18

${ }^{7}$ G. Greczynski, J. Jensen, L. Hultman, Thin Solid Films 519 (2011) 6354-6361

${ }^{8}$ K. Macak, V. Kouznetsov, J. Schneider, U. Helmersson and I.Petrov, J. Vac. Sci. Technol. A 18 (2000) 1533

9 J.T. Gudmundsson Journal of Physics: Conference Series 100 (2008) 082013

${ }^{10}$ S. M. Rossnagel, J. Vac. Sci. Technol. A 6, 19-24 (1988).

${ }^{11}$ G. Greczynski and L. Hultman, Vacuum 84 (2010) 1159-1170

${ }^{12}$ D. Lundin, N. Brenning, D. Jadernas, P. Larsson, E.Wallin, M. Lattemann, M. A. Raadu and U. Helmersson. Plasma Sources Sci. Technol. 18 (2009) 045008

${ }^{13}$ G. Greczynski, J. Lu, M. Johansson, J. Jensen, I. Petrov, J.E. Greene, and L. Hultman, Surf. Coat. Technol. 206 (2012) 4202

${ }^{14}$ G. Greczynski, J. Lu, M. Johansson, J. Jensen, I. Petrov, J.E. Greene, and L. Hultman, Vacuum 86 (2012) 1036

${ }^{15}$ G. Greczynski, J. Lu, J. Jensen, I. Petrov, J.E. Greene, S. Bolz, W. Kölker, Ch. Schiffers, O. Lemmer and L. Hultman, J. Vac. Sci. Technol. A 30 (2012) 061504

${ }^{16}$ G. Greczynski, J. Lu, J. Jensen, I. Petrov, J.E. Greene, S. Bolz, W. Kölker, Ch. Schiffers, O. Lemmer and L. Hultman, Thin Solid Films 556 (2014) 87

17 J. A. Davis, W. D. Sproul, D. J. Christie, and M. Geisler, in Proceedings of the 47th Annual SVC Technical Conference, Dallas (TX), 2004, p. 215

${ }^{18}$ D. A. Glocker, M. M. Romach, D. J. Christie, and W. D. Sproul, in Proceedings of the 47th Annual SVC Technical Conference, Dallas (TX), 2004

${ }^{19}$ W. D. Sproul, D. J. Christie, and D. C. Carter, in Proceedings of the 47th Annual SVC Technical Conference, Dallas (TX), 2004

${ }^{20}$ M. Samuelsson, D. Lundin, J. Jensen, M.A. Raadu, J.T. Gudmundsson, U. Helmersson, Surf. Coat. Technol. 205 (2010) 591

${ }^{21}$ D. J. Christie, Journal of Vacuum Science and Technology A 23 (2005) 330.

22 J. Vlcek, P. Kudlacek, K. Burcalova, and J. Musil, Jornal of Vacuum Science and Technology A 25 (2007) 42

${ }^{23}$ D. Lundin, P. Larsson, E. Wallin, M. Lattemann, N. Brenning, and U. Helmersson, Plasma Sources Sci. Technol. 17 (2008) 035021

${ }^{24}$ J. Emmerlich, S. Mraz, R. Snyders, K. Jiang, and J. M. Schneider, Vacuum 82, (2008) 867.

${ }^{25}$ A. Anders, J. Vac. Sci. Technol. A 28 (2010) 783

${ }^{26}$ U. Helmersson, M. Lattemann, J. Bohlmark, A. P. Ehiasarian, and J. T. Gudmundsson, Thin Solid Films 513 (2006) 1

${ }^{27}$ with the experimental conditions used here the average sputtered atoms mean free paths are between 3 and 6 cm, see e.g. W.D. Westwood J. Vac Sci Technol. 1978;15:1.

${ }^{28}$ in the case of HIPIMS in the industrial-size cathodes the amount of energy stored in the capacitor bank of the HIPIMS power supply is often not high enough to sustain a constant voltage during the entire pulse period due to high $I_{T}(t)$ values. In such a case, a drop in $V_{T}(t)$, that occurs with the rate directly proportional to the amplitude of the target current density, $J_{T}(t)=C / A \times d V_{T}(t) / d t$ (in which $\mathrm{C}$ is the size of the capacitor bank) takes place resulting in a triangular pulse form.

${ }^{29}$ David R. Lide (ed), CRC Handbook of Chemistry and Physics, 84th Edition. CRC Press. Boca Raton, Florida, 2003; Section 10, Atomic, Molecular, and Optical Physics; Ionization Potentials of Atoms and Atomic Ions

${ }^{30}$ G. Greczynski, I. Petrov, J.E. Greene, and L. Hultman, Vacuum 116 (2015) 36

${ }^{31}$ A. Anders Appl. Phys. Letters 92 (2008) 201501

${ }^{32}$ R. A. Baragiola, E. V. Alonso, J. Ferron, and A. Oliva-Florio, Surf. Sci. 90 (1979) 240

33 all $\phi_{T}$ values from: CRC Handbook of Chemistry and Physics version 2008, p. 12-114

${ }^{34}$ G. Greczynski, J. Lu, J. Jensen, S. Bolz, W. Kölker, Ch. Schiffers, O. Lemmer, J.E. Greene, and L. Hultman, Surf. Coat. Technol. 257 (2014) 15

${ }^{35}$ See for instance: E. P. Vaulin, N. E. Georgieva, and T. P. Martinenko, Sov. Phys. Solid State 19, 827 (1977)

${ }^{36}$ to derive Eqn.(3) we used the fact that the sputter yield $\gamma$ can be fitted with an allometric function of the form $a E_{i}^{b}$ where $a$ and $b$ are fitting parameters and ion energy $E_{i} \cong q_{n} V_{T}$ (no

${ }^{37}$ J. F. Ziegler, J. P, Biersack, U. Littmark, "The Stopping and Range of Ions in Solids," vol. 1 of series "Stopping and Ranges of Ions in Matter," Pergamon Press, New York (1984). 
${ }^{38}$ www.srim.org; accessed on 2015-06-28. 


\begin{tabular}{|c|c|c|c|c|c|c|c|c|}
\hline $\begin{array}{c}\text { Target } \\
\text { Element }\end{array}$ & $\begin{array}{c}m \\
{[\mathrm{amu}]}\end{array}$ & $\begin{array}{c}\rho \\
{\left[\mathrm{g} / \mathrm{cm}^{3}\right]}\end{array}$ & $T_{s} / T_{m}$ & $\begin{array}{c}I P_{T}^{1} \\
{[\mathrm{eV}]}\end{array}$ & $\begin{array}{c}I P_{T}^{2} \\
{[\mathrm{eV}]}\end{array}$ & $\begin{array}{c}\gamma_{\mathrm{Ar} \rightarrow \mathrm{M}} \\
\left(E_{i}=1 \mathrm{keV}\right)\end{array}$ & $\begin{array}{c}\gamma_{\mathrm{M} \rightarrow \mathrm{M}} / \gamma_{\mathrm{Ar} \rightarrow \mathrm{M}} \\
\left(E_{i}=1 \mathrm{keV}\right)\end{array}$ & $\begin{array}{c}\phi_{T} \\
{[\mathrm{eV}]}\end{array}$ \\
\hline $\mathrm{Al}$ & 26.98 & 2.70 & 0.72 & 6.01 & 18.89 & 1.08 & 1.32 & $4.1-4.3$ \\
\hline $\mathrm{Si}$ & 28.09 & 2.33 & 0.4 & 8.15 & 16.35 & 0.57 & 1.42 & $4.6-4.9$ \\
\hline $\mathrm{Ti}$ & 47.87 & 4.51 & 0.35 & 6.85 & 13.62 & 0.98 & 0.85 & 4.3 \\
\hline $\mathrm{Cr}$ & 52.0 & 7.19 & 0.31 & 6.79 & 16.54 & 2.10 & 0.89 & 4.5 \\
\hline $\mathrm{Y}$ & 88.91 & 4.47 & 0.37 & 6.24 & 12.27 & 1.30 & 0.77 & 3.1 \\
\hline $\mathrm{Zr}$ & 91.22 & 6.52 & 0.31 & 6.66 & 13.21 & 1.20 & 0.80 & 4.1 \\
\hline $\mathrm{Nb}$ & 92.91 & 8.57 & 0.24 & 6.78 & 14.35 & 1.20 & 0.76 & $4.0-4.9$ \\
\hline $\mathrm{Hf}$ & 178.49 & 13.31 & 0.27 & 6.85 & 14.98 & 1.62 & 0.86 & 3.9 \\
\hline $\mathrm{Ta}$ & 180.95 & 16.69 & 0.2 & 7.91 & 15.60 & 1.58 & 0.88 & $4.0-4.8$ \\
\hline $\mathrm{W}$ & 183.34 & 19.25 & 0.18 & 8.01 & 17.68 & 1.63 & 0.82 & $4.3-5.2$ \\
\hline
\end{tabular}

Tab. 1 


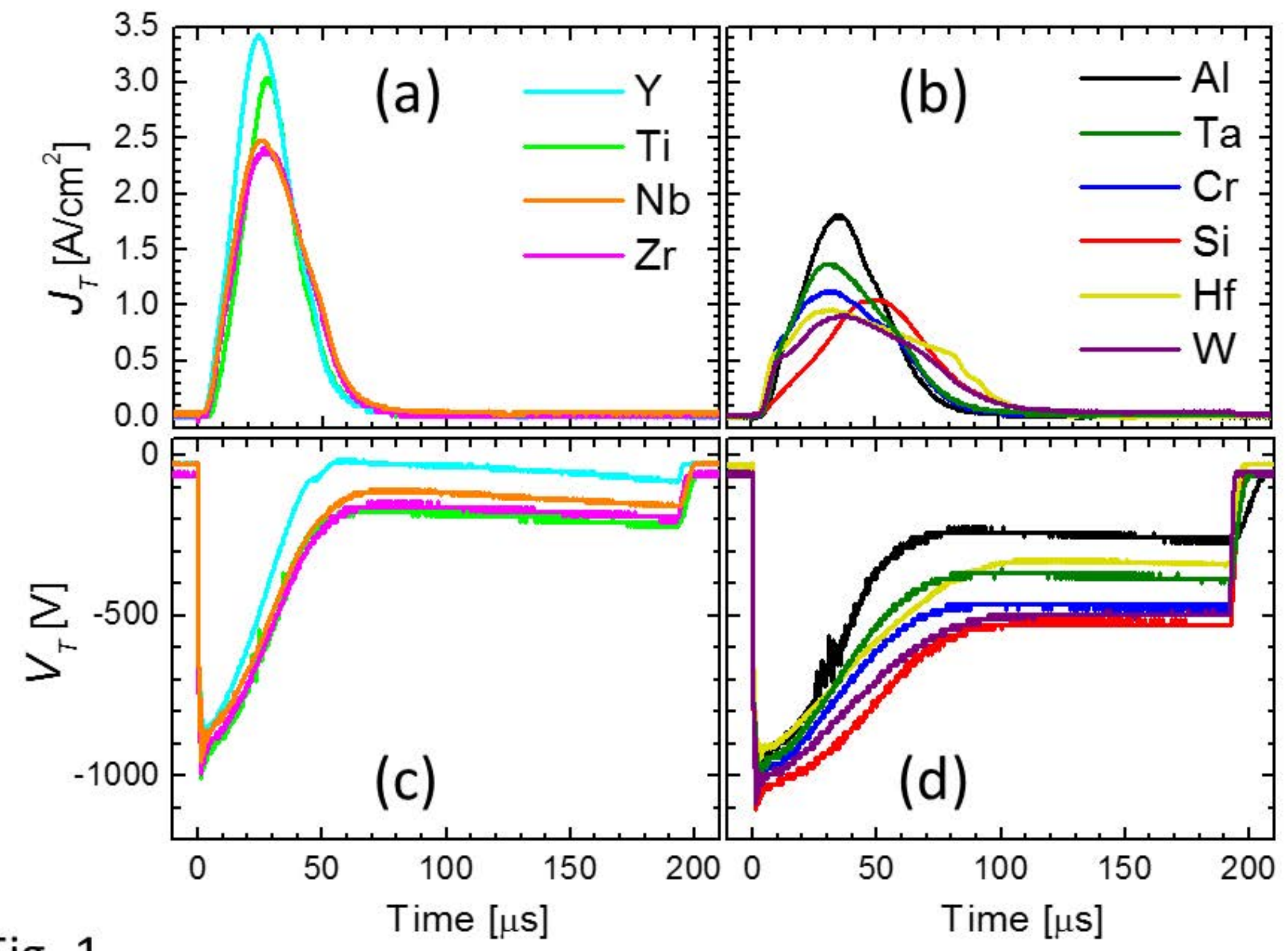

Fig. 1 


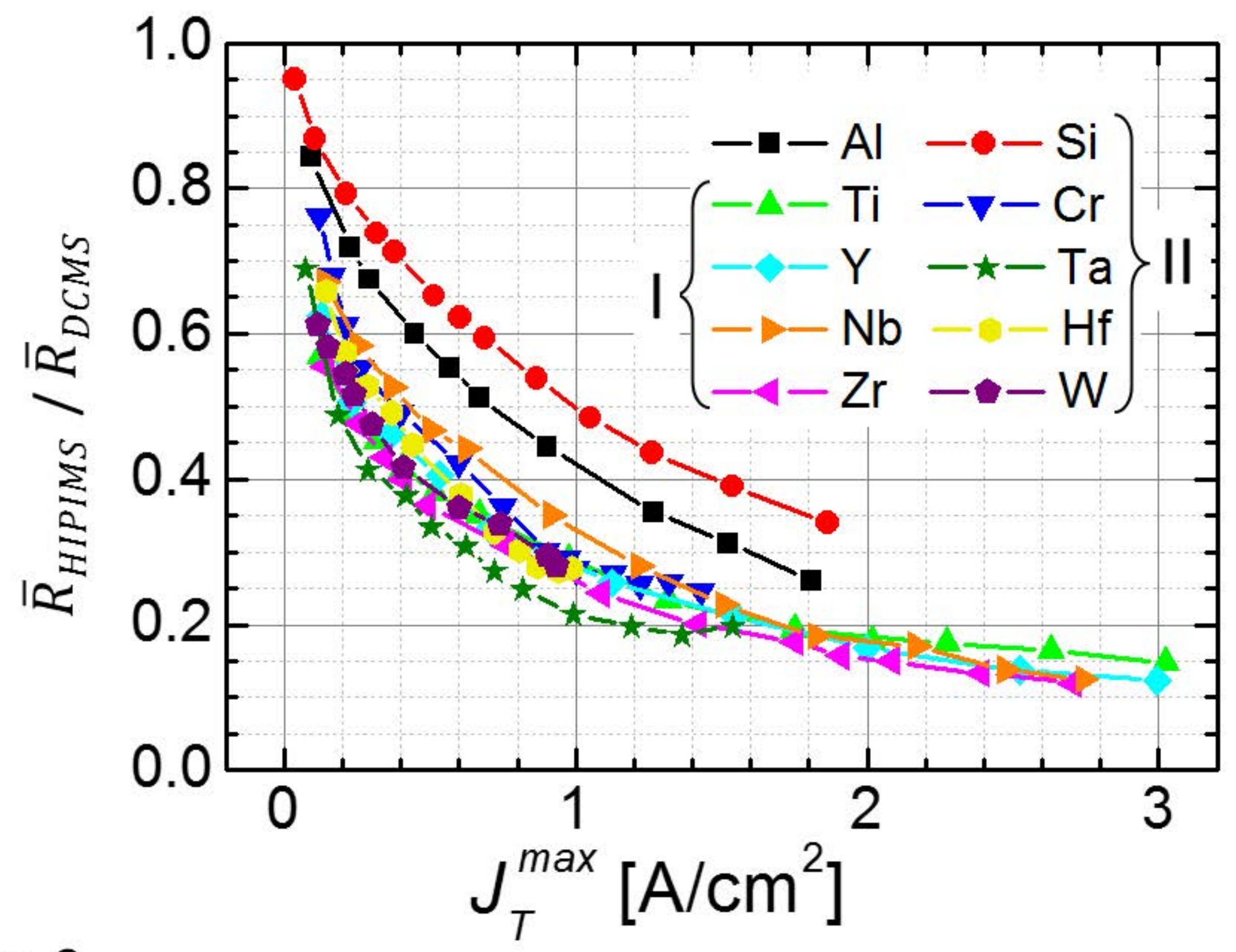

Fig. 2 